\title{
REGULARIZED SUM FOR EIGENFUNCTIONS OF MULTI-POINT PROBLEM IN THE COMMENSURABLE CASE
}

\author{
S.A. SALEH \\ Department of Mathematics \\ Faculty of Science \\ Tanta University \\ Tanta, Egypt \\ (Received January 3, 1995 and in revised form May 22, 1995)

$$
-y^{\prime \prime}(x)+q(x) y(x)=\lambda y(x) ; \quad 0 \leq x \leq \pi
$$

$$
\begin{aligned}
& U_{1}(y)=\alpha_{1} y(0)+\alpha_{2} y(\pi)+\sum_{k=3}^{n} \alpha_{k} y\left(x_{k} \pi\right)=0 \\
& U_{2}(y)=\beta_{1} y(0)+\beta_{2} y(\pi)+\sum_{k=3}^{n} \beta_{k} y\left(x_{k} \pi\right)=0
\end{aligned}
$$

ABSTRACT. Consider the eigenvalue problem which is given in the interval $[0, \pi]$ by the differential equation

and multi-point conditions

where $q(x)$ is sufficiently smooth function defined in the interval $[0, \pi]$. We assume that the points $x_{3}, x_{4}, \ldots, x_{n}$ divide the interval $[0,1]$ to commensurable parts and $\alpha_{1} \beta_{2}-\alpha_{2} \beta_{1} \neq 0$. Let $\lambda_{k, s}=\rho_{k, s}^{2}$ be the eigenvalues of the problem (0.1)-(0.2) for which we shall assume that they are simple, where $k, s$, are positive integers and suppose that $H_{k, s}(x, \xi)$ are the residue of Green's function $G(x, \xi, \rho)$ for the problem (0.1)-(0.2) at the points $\rho_{k, s^{\circ}}$. The aim of this work is to calculate the regularized sum which is given by the form :

$$
\sum_{(k)} \sum_{(s)}\left[\rho_{k, s}^{-\sigma} H_{k, s}(x, \xi)-R_{k, s}(\sigma, x, \xi, \rho)\right]=S_{\sigma}(x, \xi)
$$

The above summation can be represented by the coeflicients of the asymptotic expansion of the function $G(x, \xi, \rho)$ in negative powers of $k$. In series $(0.3) \sigma$ is an integer, while $R_{k .}(\sigma, x, \xi, \rho)$ is a function of variables $x, \xi$ and defined in the square $[0, \pi] x[0, \pi]$ which ensure the convergence of the series (0.3).

KEY WOREISS ANI) PIIRASES : Regularized sum for cigenfunctions, asymptotic formula, Green's function, differential operator.

1991 AMS SUB.JECT C.LASSIFICATION CODES : 47F05

\section{INTROJUCTION.}

It is well-known that the sum of the diagonal elements in a square matrix is equal to the sum of the eigenvalues of its operator in finite dimensional space. In other words the trace of a matrix is equal to the spectral trace in n-rlimensional spaces. 
It is worth mentioning that this theorem is satisfied also in the case of nuclear operators acting in Hilbert space. Sadovnichii [1] proved this theorem. Thus we might ask the following question. Is the last theorem applicable to the case of unbounded operators?, especially in the case of differential operators since in general case the trace of a matrix and spectral trace do not exist. Consider, for example, the boundary-value problem :

$$
\begin{aligned}
& -y^{\prime \prime}(x)+q(x) y(x)-\lambda y(x), \quad 0 \leq x \leq \pi \\
& y(0)=y(\pi)=0,
\end{aligned}
$$

where $q(x)$ is sufficiently smooth function.

The eigenvalues $\lambda_{n}$ of problem (1.1), (1.2) has the asymptotic expausion in the form :

$$
\lambda_{n} \sim n^{2}+c_{0}+\frac{c_{2}}{n^{2}}+\frac{c_{4}}{n^{4}}+\ldots
$$

where

$$
c_{0}=\frac{1}{2 \pi} \int_{0}^{\pi} q(\xi) d \xi
$$

From Equation (1.3) it is clear that $\sum_{n=1}^{\infty} \lambda_{n}$ diverges, while $\sum_{n=1}^{\infty}\left(\lambda_{n}-n^{2}-c_{0}\right)$ converges, and is called the regular trace for the problem (1.1), (1.2).

The study of regular trace for differential operators plays an important role in several fields such as mathematical analysis, theoretical physics and quantum mechanics, where the regular traces give the asymptotic expansion for the cigenvalues of operators. We can also use the regular trace in the inverse spectral problems in functional analysis.

A good number of works has been devoted to the deduction of the formulae of regularized traces of differential operators Gelfand, I,evitian [2], Charles, Halberg and Kramer [3], Lidsky, Sadovnichii [4, 5, 6], Sadovnichii, Iyubishkin and Belabbasy [7, 8], Saleh [9] and many other authors

The concept of the regularized trace with a weight for the differential operators was introduced by Sadovnichii [10].

The main goal now is to derive asymptotic formulae for the solutions of $(0.1)$ when $|\lambda| \rightarrow \infty$ and then use them to obtain the asymptotic formulae for the eigenvalues of the problem (0.1), (0.2). The concluding part of this paper is devoted to the derivation of the regularized sums of eigenfunctions of the second order, and we shall give some examples, to illustrate the mentioned concept of regularized sums of eigenfunctions.

\section{ASYMPTOTIC, FORMI,AE, FOR THE SOI,UTION OF THE STURM-LIOUVILLE EQUATION}

The solution of the differential equation (0.1) admits asymptotic expansions in powers of $\rho^{-1}$ which become more precise as the number of derivatives that the function $q(x)$ has increases " Marchenko [11], Naimark [12]. Let $y_{1}(x, \rho)$ and $y_{2}(x, \rho)$ be linearly independent solutions of (0.1), then

$$
\begin{aligned}
& y_{1}(x, \rho)=e^{i \rho \rho x}\left[1+\sum_{v=1}^{N} \frac{u_{v}(x)}{\rho v}+O\left(-\frac{1}{\rho N+1}\right)\right] \text {, } \\
& y_{2}(x, \rho)=e^{-i \rho x}\left[1+\sum_{v=1}^{N} \frac{(-1)^{v} u_{v}(x)}{\rho^{v}}+O\left(\frac{1}{\rho N+1}\right)\right] \text {, }
\end{aligned}
$$

where $N$ positive integer depends on the smoothness of the function $q(x)$ and the functions $u_{v}(x)$, $v=1,2, \ldots, N$ admit the representations: 


$$
\begin{aligned}
& u_{1}(x)=\frac{1}{2 i} \int_{2}^{x} q(\xi) d \xi, \\
& u_{2}(x)=\frac{1}{4}\left[q(x) \cdots q(0)-\frac{1}{2}\left(\int_{0}^{x} q(\xi) d \xi\right)^{2}\right], \\
& u_{v+2}(x)=\frac{1}{2 i} \int_{0}^{x}\left\{-d^{2}+q(\xi)\right\} u_{v+1}^{2}(\xi) d \xi ; \quad v=1,2,3, \ldots, N-2
\end{aligned}
$$

We note that $u_{v}(0):=0$, for $v:=1,2, \ldots, N$. By means of the asymptotic formulae (2.1) and Equation (2.2) we can prove that

$$
\begin{aligned}
& \delta(x, \rho)=\delta(0, \rho):\left.W\left[y_{1}, y_{2}\right]\right|_{x=0} \\
& =-2 i \rho+i\left[\frac{q(0)}{2 p}-\frac{q^{\prime \prime \prime}(0)-4 q(0) q^{\prime}(0)}{4 \rho^{3}}+\ldots\right]
\end{aligned}
$$

\section{ASYMPTOTIC FORMULAE FOR FIGENVAI,IES OF THE PROBI,EM (0.1)-(0.2) IN THE} COMMENSURABIE C:ASE:

In Saleh [9] proved that the eigenvalues of the problem (0.1)-(0.2) $\left(\lambda=\rho^{2}\right)$ are found from the condition :

where

$$
\begin{aligned}
& f(p)=\frac{\Lambda(p)}{\tilde{\delta}(p)}=0, \\
& \Delta(\rho)=\operatorname{det}\left\|U_{i}\left(y_{k}\right)\right\|_{i, k=1}^{2}
\end{aligned}
$$

Upon using Equations (2.1), (2.2) it is easy to see that

$$
\begin{aligned}
& \Delta(\rho)=\sum_{k=1}^{2 n^{2}-6} A_{k}(\rho)\left[\gamma_{k}^{(0)}+\frac{\beta_{k}^{(1)}}{\rho}+\frac{\beta_{k}^{(2)}}{\rho^{2}}+\ldots+\frac{\beta_{k}^{(N)}}{\rho^{N}}+\ldots\right], \\
& A_{k}(\rho)=e^{i \xi_{k} \pi \rho}, \xi_{1}=-1, \xi_{2}=-\left(1-x_{3}\right), \xi_{2 n^{2}-6}=-\xi_{1}=1 \text {, }
\end{aligned}
$$

where and $\gamma_{k}^{(0)}, \beta_{k}^{(j)},(j=1,2,3, \ldots)$ arc calculated in terms of the constants $\alpha_{v}, \beta_{v}(v=1,2, \ldots, n)$ and the function $q(x)$, for example,

$$
\begin{aligned}
& \gamma_{1}^{(0)}=\left(\alpha_{1} \beta_{2}-\beta_{1} \alpha_{2}\right)=-\gamma_{2 n^{2}-6}^{(0)}, \\
& \beta_{1}^{(1)}=-\beta_{2 n^{2}-6}^{(1)}=\frac{1}{2 i}\left(\beta_{1} \alpha_{2}-\alpha_{1} \beta_{2}\right) \int_{0}^{\pi} q(\xi) d \xi
\end{aligned}
$$

Using the results of Saleh [9], we deduce that in commensurable case the problem (0.1)-(0.2) has $\mathbf{2 m}$ series of eigenvalues which have the fọllowing asymptotic formula:

$$
\rho_{k, s} \sim 2 m k-\frac{m}{i \pi} \ln a_{0}^{(s)}+\frac{a^{(s)}}{2 a_{0}^{(s)} k} \frac{a_{1}^{(s)} \ln a_{0}^{(s)}}{4 i a_{0}^{(s)} \pi k^{2}}+\ldots,
$$

where

$$
\mathbf{m}=\frac{1}{d} ; \quad d=\min \left\{\xi_{1}, \xi_{2}, \xi_{3}, \ldots, \xi_{2 n^{2}-6}\right\}
$$

4. THE ASYMPTOTIC FOIRMIAE FOR IIIF GIRFEN'S FINC'IION OF THE PROBLEM (0.1)-(0.2) IN COMMENSURABILE C.ASE:

It is well-known that the Green's function of differential equation of the second order is given by the formula: 
where

$$
G(x, \xi, \rho)=\frac{1}{\Delta(\rho)}\left|\begin{array}{ccc}
y_{1}(x, \rho) & y_{2}(x, \rho) & g(x, \xi, \rho) \\
U_{1}\left(y_{1}\right) & U_{1}\left(y_{2}\right) & U_{1}(g) \\
U_{2}\left(y_{1}\right) & U_{2}\left(y_{2}\right) & U_{2}(g)
\end{array}\right|
$$

( The positive sign being taken if $x>\xi$, and the negative sign if $x<\xi$ ).

If we divide the $\rho$-plane into four regions $\tilde{\mathrm{S}}_{0}, \tilde{\mathrm{S}}_{1}, \tilde{\mathrm{S}}_{2}, \tilde{\mathrm{S}}_{3}$ such that:

$$
\begin{aligned}
& \tilde{\mathbf{S}}_{0}=\left\{\rho:|\rho|>\mathbf{R}, \quad 0<\arg \rho \leq \frac{\pi}{2}-\theta\right\} \text {, } \\
& \tilde{S}_{1}=\left\{\rho:|\rho|>R, \quad \frac{\pi}{2}-\theta<\arg \rho \leq \pi\right\} \text {, } \\
& \tilde{\mathbf{S}}_{2}=\left\{\rho:|\rho|>\mathbf{R}, \pi<\arg \rho \leq \frac{3 \pi}{2}-\theta\right\} \text {, } \\
& \tilde{S}_{3}=\left\{\rho:|\rho|>R, \quad \frac{3 \pi}{2}-\theta<\arg \rho \leq 2 \pi\right\},
\end{aligned}
$$

we see that the Green's function of the problem $(0.1)-(0.2)$ in the commensurable case has the following asymptotic formula:

where

$$
\begin{aligned}
& G(x, \xi, \rho) \sim e^{i(x-\xi) \rho} \sum_{v=0}^{\infty} \frac{\phi^{(1)}}{\rho^{v}} ; \rho \in \tilde{S}_{0}^{\delta}, \xi<x<x_{j} \pi, \\
& G(x, \xi, \rho) \sim e^{-i(x, \xi) \rho} \sum_{v=0}^{\infty} \frac{\phi^{(2)}}{\rho^{v}} ; \rho \in \tilde{S}_{0}^{\delta} ; \xi<x_{j} \pi<x,
\end{aligned}
$$

and,

$$
\tilde{\mathbf{S}}_{\mathbf{0}}^{\delta}=\tilde{\mathbf{S}}_{\mathbf{0}} \backslash Q_{\mathbf{k}, \mathbf{s}}^{\delta}
$$

$$
Q_{k, s}^{\delta}=\left\{\rho:\left|\rho-\rho_{k, s}\right| \leqslant \delta, \Delta\left(\rho_{k, s}\right)=0\right\}
$$

Since $H_{k, s}(x, \xi)$ the residue of Green's function $G(x, \xi, p)$ for problem $(0.1)-(0.2)$ in the points $\rho_{k, s}$ and from the assumption that the eigenvalues $\rho_{k, s}$ are simple, then

$$
H_{k, s}\left(x, \xi, \rho_{k, s}\right)=\lim _{\rho \rightarrow \rho_{k, s}}\left(\rho-\rho_{k, s}\right) G(x, \xi, \rho)
$$

Upon using the asymptotic formulae(4.4) for $G(x, \xi, \rho)$ in $\bar{S}^{\delta}$ and Equation (4.5) we have for $H_{k, s}(x, \xi)$ the following asymptotic formulae

$$
\begin{aligned}
& H_{k, s}\left(x, \xi, \rho_{k, s}\right) \sim e^{i(x-\xi) \rho_{k, s}} \sum_{v=0}^{\infty} \frac{\phi_{0, j}^{(3)}}{v}, \rho \in \tilde{\Gamma}_{k, s}^{v} \in, \xi<x<x, \pi \\
& H_{k, s}\left(x, \xi, \rho_{k, s}\right) \sim e^{-i(x-\xi) \rho_{k, s}} \sum_{\nu}^{\infty} \frac{\phi_{0, j}^{(4)}}{\rho_{k, s}^{\prime \prime}}, \rho \in \tilde{S}_{0}^{\delta}, \quad \xi<x_{j} \pi<x,
\end{aligned}
$$

where the functions $\phi_{v, j}^{(1)}, \phi_{v, j}^{(2)}, \phi_{v, j}^{(3)}$ and $\underset{v, j}{(4)}$ are defined in terms of the constants 
$\alpha_{k}, \beta_{k},(k=1,2, \ldots, n)$ and the function $q(x), j=3,4,5, \ldots, n$. We note that for the functions $\Delta(p)$, $\Delta^{\prime}(\rho)$ have asymptotic formulae:

$$
\begin{aligned}
& \Delta(\rho)_{\sim e^{-i \rho \pi}} \sum_{v=0}^{\infty} \frac{\Psi_{U}^{(1)}}{\rho^{U}}, \quad \rho \in \tilde{S}_{0}^{\delta} \\
& \Delta^{\prime}(\rho)_{\sim e^{-i \rho \pi}} \sum_{U=0}^{\infty} \frac{\Psi_{v}^{(2)}}{\rho^{v}}, \quad \rho \in \tilde{S}_{0}^{\delta}
\end{aligned}
$$

\section{REGLARIZED SUM FOR EIGENFUNCTIONS OF THE PROBLEM (0.1)-(0.2) IN THE COMMENSURABLE CASE}

Now we wish to evaluate the functions $R_{k, s}(\sigma, x, \xi)$ which ensure the convergence of the series(0.3). We must first estimate the functions $\rho_{k, s}^{-\sigma} H_{k, s}(x, \xi)$ in $\tilde{s}_{0}^{\sigma}$. From Equation (3.5), we have

$$
\rho_{\mathbf{k}, \mathbf{s}}^{-\sigma} \sim \sum_{u=0}^{\infty} \frac{\mathbf{Q}_{v}^{(\mathbf{s})}(\sigma)}{\mathbf{k}^{\mathrm{v}+\sigma}}
$$

In the asymptotic formula (5.1)

$$
\begin{aligned}
& Q_{0}^{(s)}(\sigma)=(-2 m)^{-\sigma}, \quad Q_{1}^{(s)}(\sigma)=(-1)^{-\sigma+1}(2 m)-\sigma-1 \frac{m}{i \pi} \ln a_{0}^{(s)}, \\
& Q_{2}^{(s)}(\sigma)=(-2 m)^{-\sigma}\left[\left(\begin{array}{c}
-\sigma \\
1
\end{array}\right) \frac{a_{1}^{(s)}}{2 a_{0}^{(s)}(-2 m)}+\left(\begin{array}{c}
-\sigma \\
2
\end{array}\right) \frac{\left(\ln a_{0}^{(s)}\right)^{2}}{4 \pi^{2}}\right], \ldots
\end{aligned}
$$

From Equation (3.5) we get

$$
e^{i \rho_{k, s}(x-\xi)} \sim e^{i(x-\xi)\left(-2 m k-m_{i \pi} \ln a_{0}^{(s)}\right.} \sum_{n=0}^{\infty} \frac{\Psi_{n}^{(s)}(x, \xi)}{k^{n}},
$$

where the functions $\psi_{n}^{(s)}(x, \xi)$ are polynomials of $(x-\xi)$.

Upon using Equations(4.5), (5.1) and (5.2), we have

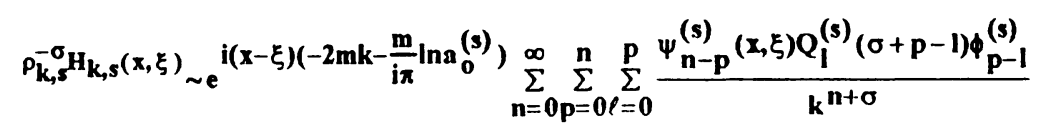

For large number $\tau$, we consider the function

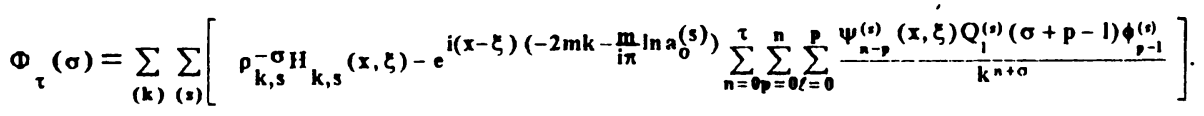

It is clear that the function $\Phi \tau(\sigma)$ may be extended to analytic function in the half plane $\operatorname{Re} \sigma>-\tau$

THEOREM 5.1 If $\operatorname{Re} \sigma>-\tau$ then

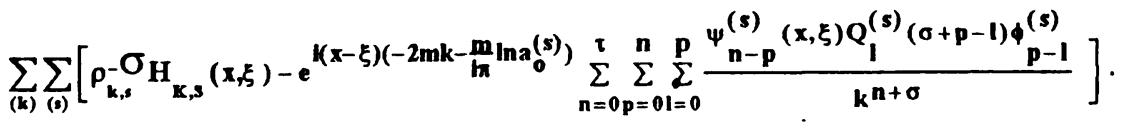

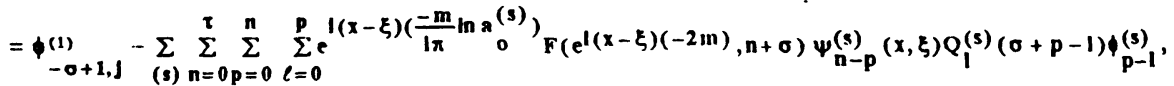

where

$$
F(x, \sigma)=\sum_{k=1}^{\infty} \frac{z^{k}}{k^{\sigma}}
$$


THEOREM 5.2 if $\sigma=-2$, we have

$$
\begin{aligned}
& \left.\sum_{(k)(s)} \sum_{k, s} H_{k, s}(x, \xi)-e^{1(x-\xi)\left(-2 m k-\frac{m}{l n} \ln a_{0}^{(s)}\right)} \sum_{n=0}^{3} \sum_{p=0}^{n} \sum_{\ell=0}^{p} \frac{\Psi_{n-p}^{(s)}(x, \xi) Q_{1}^{(S)}(-2+p-1) \Phi_{p-1}^{(s)}}{k^{n-2}}\right] \\
& =\phi_{3,1}^{(1)}-\sum_{(s) n=0} \sum_{p=0} \sum_{p}^{n} e^{i(x-\xi)\left(-\frac{m}{i \pi} \ln a^{(s)}\right.} F\left(e^{i(x-\xi)(-2 m)}, n-2\right) \Psi_{n-p}^{(s)} Q_{\ell}^{(s)} \phi_{p-\ell}^{(s)} .
\end{aligned}
$$

REMARK. From the definition of $F(x, \sigma)$ it is clear that this function satisfies the following properties

1. $F(z, \sigma)=z \Phi(z, \sigma, 1)$, where

$$
\Phi(z, \sigma, v)=\sum_{n=0}^{\infty}(v+n)^{-\sigma} z^{n}=\frac{1}{\Gamma(\sigma)} \int_{0}^{\infty} \frac{t^{\sigma-1} e^{-(v-1) t}}{e^{t}-z} d t,
$$

$\operatorname{Re} v>0$ and either $|z| \leq 1, \quad z \neq 1, \operatorname{Re} \sigma>0$ or $z=1, \operatorname{Re} \sigma>1$.

2. $F(z,-m)=(-1)^{m+1} F\left(\frac{1}{z},-m\right), m=1,2,3, \ldots$

3. $F(z, s)+e^{i s \pi} F\left(\frac{1}{z}, s\right)=\frac{(2 \pi)^{s}}{\Gamma(s)} e^{i \pi s / 2} \zeta\left(1-s, \frac{\log z}{2 \pi i}\right)$

4. Equations (0.2), (0.3) furnish the analytical continuation of the series $\sum_{n=1}^{\infty} z^{n}$ beyond the circle of convergence $|z|=1$

If $F_{0}(z)$ denotes the principal branch of $F(z)$ in the cut $z$-plane $[0<\arg (z-1)<2 \pi]$, the cut being imposed from 1 to $\infty$ along the real axis, the difference of the values of $F_{0}(z)$. between a point on the upper edge of the cut and a point on the lower edge, according to (0.3),

$$
F_{0}(x, s)-F_{0}\left(x e^{2 i \pi}, s\right)=2 \pi i(\log x)^{s-1} / \Gamma(s)
$$

Hence, if we cross the cut, from the upper half-plane to the lower half-plane, we obtain for the continuation $F_{1}(z)$ of $F_{0}(z)$

$$
F_{1}(z)=F_{0}(x)+2 \pi i(\log x)^{s-1} / \Gamma(s)
$$

The analogous formula for the inverse process of continuation is

$$
F_{2}(z)=F_{0}(z)-2 \pi i(\log z)^{s-1} / \Gamma(s)
$$

5. $F\left(e^{i t},-m\right)=\left(i \frac{d}{d t}\right)^{m} \frac{1}{e^{-i t}-1} ; m=1,2,3 \ldots$

The previous properties of $F(x, \sigma)$ are proved in A. Eredelyi, W. Magnus, F.

Oberhettinger and F. G. Tricomi [13]

6. D. Klusch [14] considered the generalized zeta function in the from

$$
L(x, a, s)=\sum_{n \geq 0}(2 \pi i n x)(n+a)^{-s}
$$

( $a \in R^{+} ; x$ is not integer, $\operatorname{Re} s>0$; and if $x$ is an integer, $\operatorname{Re} s>1$ )

and studied some further properties of the function $L(x, n, s)$ resulting from the taylor expansion of the function $W(\zeta)=L(x, a+\zeta, s)$ in the neighbourhood $\xi=0$

Now, we consider the following examples:

6. EXAMPIES

1. Consider the problem

$$
\begin{aligned}
& -y^{\prime \prime}(x)=\lambda y(x) \quad 0 \leq x \leq \pi, \\
& y(0)=y(\pi)=0
\end{aligned}
$$


Its clear that the eigenvalues of problem (6.1), (6.2) are $\lambda_{n}=n^{2}$ and the corresponding eigenfunctions are $y_{n}(x)=\sin n x$, so the regularized trace of problem (6.1), (6.2) is $\sum_{n=1}^{\infty}\left(\lambda_{n}-n^{2}\right)=0$, and the regularized sum of eigenfunctions of problem (6.1)-(6.2) is given by the following formula

$$
\sum_{(n)}\left[\lambda_{n} H_{n}(x, \xi)+\frac{n}{\pi} \sin n x \sin n \xi\right]=0 .
$$

2. Consider the Sturm Liouville problem

$$
\begin{aligned}
& -y^{\prime \prime}(x)+q(x) y(x)=\lambda y, \quad \lambda=p^{2}, \quad 0 \leq x \leq \pi \\
& y(0)=y(\pi)=0,
\end{aligned}
$$

where $q(x)$ is a sufficiently smooth function defined in the interval $[0, \pi]$.

Let $y_{1}(x, \rho), y_{2}(x, \rho)$ are two independant solutions of Equation (6.4) such that

$$
y_{j}^{(k-1)}(0, \rho)= \begin{cases}1 & k=j \\ 0 & k \neq j\end{cases}
$$

Then from Equations (2.1), (2.2) and (2.3), we have

$$
\begin{aligned}
& y_{1}(x, \rho)=\sum_{v=0}^{N} \frac{A_{v}(x, \rho)}{\rho^{v}}+O\left(\frac{1}{\rho+1}\right) \\
& y_{2}(x, \rho)=\sum_{v=1}^{N} \frac{B_{v}(x, \rho)}{\rho^{v}}+O\left(\frac{1}{\rho+1}\right)
\end{aligned}
$$

where

$$
\begin{aligned}
& A_{0}(x, \rho)=\cos \rho x \\
& A_{1}(x, \rho)=i u_{1}(x) \sin \rho x, \\
& A_{2}(x, \rho)=u_{2}(x) \cos \rho x \\
& A_{3}(x, \rho)=i\left[u_{3}(x)+i u_{2}^{1}(0)\right] \sin \rho x, \ldots \\
& B_{1}(x, \rho)=\sin \rho x \\
& B_{2}(x, \rho)=-i u_{1}(x) \cos \rho x \\
& B_{3}(x, \rho)=-i\left(i u_{2}(x)-u_{1}^{1}(0)\right) \sin \rho x, \ldots \\
& B_{4}(x, \rho)=\left[2 u_{1}(x) u_{1}(0)-i u_{3}(x)\right] \cos \rho x, \ldots
\end{aligned}
$$

and $N$ is a positive integer depending on the smoothness of the function $q(x)$.

Since $\Delta(\rho)=\operatorname{det}\left\|U_{j}\left(y_{k}\right)\right\|_{j, k}^{2}$, then

$$
\Delta(\rho)=y_{2}(\pi, \rho)=\sum_{v=1}^{N} \frac{B_{v}(\pi, \rho)}{\rho^{v}}+O\left(\frac{1}{\rho+1}\right)
$$

From the last formula, we can obtain the roots of the function $\Delta(\rho)$ which are eigenvalues of the problem (6.4)-(6.5). Upon using the successive approximation, we get the following asymptotic formula for the zeros of the function $\Delta(\rho)$ :

$$
\rho_{n} \sim n+\frac{c}{n}=\frac{1}{n}+\frac{c-3}{3}+\ldots
$$

where

$$
c_{-1}=\frac{i}{\pi} u_{1}(x), c_{-3}=\frac{1}{2 \pi i}\left[3\left\{u_{1}(\pi) u_{2}(x)-i u_{1}(\pi) u_{1}^{\prime}(0)-u_{3}(\pi)\right\}-u_{1}^{3}(x)\right], \ldots
$$


Then

where

$$
\lambda_{n \sim n^{2}}+c_{0}+\frac{c_{2}}{n^{2}} ; \frac{c_{4}}{n^{4}}+\ldots
$$

In the paper [2] I. M. Gelfand, B. M. Levitan have proved that

$$
\sum_{n=1}^{\infty}\left(\lambda_{n}-n^{2}-c_{0}\right)=\frac{1}{2} c_{0}-\frac{1}{4}[q(0)+q(\pi)]
$$

Upon using the results in H.F. Weinberger[14], we deduce that the Green's function of the problem (6.4)-(6.5) is given by the following formula:

$$
G(x, \xi, \rho)= \begin{cases}\frac{y_{2}(\xi, \rho)}{y_{2}(\pi, \rho)}\left[y_{1}(x, \rho) y_{2}(\pi, \rho)-y_{2}(x, \rho) y_{1}(\pi, \rho)\right] & x \geq \xi \\ \frac{y_{2}(x, \rho)}{y_{2}(\pi, \rho)}\left[y_{1}(\xi, \rho) y_{2}(\pi, \rho)-y_{2}(\xi, \rho) y_{1}(\pi, \rho)\right] & x \leq \xi\end{cases}
$$

From the definitions of $H_{k}\left(x, \xi, \rho_{k}\right)$, we deduce that

$$
H_{k}\left(x, \xi, \rho_{k}\right)=\left\{\begin{array}{l}
\frac{y_{2}\left(\xi, \rho_{k}\right)\left[y_{1}\left(x, \rho_{k}\right) y_{2}\left(\pi, \rho_{k}\right)-y_{2}\left(x, \rho_{k}\right) y_{1}\left(\pi, \rho_{k}\right)\right]}{y_{2}^{\prime}\left(\pi, \rho_{k}\right)} \\
\frac{y_{2}\left(x, \rho_{k}\right)\left[y_{1}\left(\xi, \rho_{k}\right) y_{2}\left(\pi, \rho_{k}\right)-y_{2}\left(\xi, \rho_{k}\right) y_{1}\left(\pi, \rho_{k}\right)\right]}{y_{2}^{\prime}\left(\pi, \rho_{k}\right)}, x \leq \xi
\end{array}\right.
$$

where $y_{2}^{\prime}\left(\pi, \rho_{k}\right)=\left.\frac{d}{d \rho}\left[y_{2}(\pi, \rho)\right]\right|_{\rho=\rho_{k}}$

Substituting Equations (6.6), (6.7) and (6.8) in (6.13), (6.14), we get the following asymptotic formulae:

$$
G(x, \xi, \rho) \sim\left\{\begin{array}{cc}
e^{i \rho(x-\xi)} \sum_{v=0}^{\infty} \frac{\varphi_{v}^{(1)}(x, \xi)}{\rho^{v}} ; & x>\xi \\
e^{-i \rho(x-\xi)} \sum_{v=0}^{\infty} \frac{\Phi_{v}^{(2)}(x, \xi)}{\rho^{v}} ; & x \leq \xi
\end{array}\right.
$$

and

$$
H_{k}\left(x, \xi, \rho_{k}\right) \sim\left\{\begin{array}{cc}
e^{i \rho(x-\xi)} \sum_{v=0}^{\infty} \frac{\varphi_{v}^{(3)}(x, \xi)}{\rho_{k}^{v}}, & x>\xi \\
e^{-i \rho(x-\xi)} \sum_{v=0}^{\infty} \frac{\phi_{v}^{(4)}(x, \xi)}{\rho_{k}^{v}} ; & x \leq \xi
\end{array}\right.
$$

In formulae (6.15) and (6.16), the functions $\varphi_{j}^{(k)}(x, \xi) \quad(k=1,2,3,4 . j=0,1,2,3, \ldots)$ can be expressed in terms of the potential $q(x)$ and its derivatives. For example:

$$
\begin{gathered}
\varphi_{0}^{(1)}=\varphi_{0}^{(2)}=0, \quad \varphi_{1}^{(1)}=\varphi_{1}^{(2)}=\frac{1}{2 i}, \quad \varphi_{2}^{(1)}=\frac{i}{2}\left(u_{1}(x)-u_{1}(\xi)\right), \quad \varphi_{2}^{(2)}=\frac{i}{2}\left(u_{1}(\xi)-u_{1}(x)\right), \\
\varphi_{3}^{(2)}=\varphi_{3}^{(1)}=\frac{i}{2}\left[u_{2}(x)+u_{1}(\xi) u_{1}(x)+u_{2}(\xi)+i_{1}^{\prime}(0)\right], \ldots
\end{gathered}
$$




$$
\begin{aligned}
& \varphi_{0}^{(3)}=\varphi_{0}^{(4)}=0, \quad \varphi_{1}^{(3)}=\varphi_{1}^{(4)}=\frac{-1}{2 \pi}, \varphi_{2}^{(3)}=\frac{1}{2 \pi}\left[u_{1}(x)-u_{1}(\xi)\right], \varphi_{2}^{(4)}=\frac{1}{2 \pi}\left[u_{1}(\xi)-u_{1}(x)\right] \ldots \\
& \phi_{3}^{(3)}=\frac{1}{2 \pi}\left[u_{2}(x)+u_{2}(\xi)-u_{1}(x) u_{1}(\xi)+i u_{1}^{\prime}(0)+\frac{i}{\pi}\left(u_{1}(x)+u_{1}(\pi)-u_{1}(\xi)-2 u_{1}^{\prime}(0)\right)-\frac{1}{\pi^{2}}\right]
\end{aligned}
$$

Using the asymptotic formula (6.9) we have

$$
\begin{aligned}
& \rho_{k}^{2}=\sum_{v=0}^{\infty} \frac{Q_{v}}{k^{2}} \\
& \text { where } \quad Q_{0}=1 \quad Q_{1}=0 \quad Q_{2}=2 C_{-1} \quad Q_{3}=0, \quad Q_{4}=2 C_{-3}
\end{aligned}
$$

When we deal with the problem (6.1)-(6.2), the formula (5.3) takes the form :

$$
\mathrm{e}^{\mathrm{I} p_{\mathrm{k}}(\mathrm{x}-\xi)} \sim \mathrm{e}^{\mathrm{kk}(\mathrm{x}-\xi)} \sum_{\mathrm{n}=0}^{\infty} \frac{\Psi_{\mathrm{n}}(\mathrm{x}, \xi)}{k^{\mathrm{n}}} .,
$$

where

$$
\begin{aligned}
& \Psi_{0}(\mathrm{x}, \xi)=1 \quad \Psi_{1}(\mathrm{x}, \xi)=\mathrm{iC}_{-1}(\mathrm{x}-\xi) \\
& \Psi_{2}(x, \xi)=-\frac{1}{2} C_{-1}^{2}(x-\xi)^{2} \quad \Psi_{3}=i C_{-3}(x-\xi)+\frac{\left(i C_{-1}(x-\xi)\right)^{3}}{6}, \ldots
\end{aligned}
$$

From the formulae (6.18), (6.19), we have

$$
\lambda_{k} H_{k}(x, \xi) \sim\left\{\begin{array}{cc}
e^{n(x-\xi)} \sum_{n=0}^{\infty} \sum_{p=0}^{\infty} \sum_{\ell=0}^{p} \frac{\Psi_{n p} Q_{\ell} \varphi_{p-\ell}^{(3)}}{k^{n-2}} & x>\xi \\
e^{-\mathbf{k}(x-\xi)} \sum_{n=0}^{\infty} \sum_{p=0}^{n} \sum_{\ell=0}^{p} \frac{\Psi_{n-p} Q_{\ell} \varphi_{p-\ell}^{(4)}}{k^{n-2}}, & x \leq \xi
\end{array}\right.
$$

Using formulae (6.17), (6.18), (6.20) and (6.21), we have the following theorem.

THEOREM 6.1 For the problem (6.1)-(6.2), the regularized sum for the eigenfunctions is given by the following formulae :

(1) If $x>\xi$, then :

$$
\begin{aligned}
& \sum_{k=1}^{\infty}\left[\lambda_{k} H_{k}(x, \xi)+\frac{1}{2 \pi} e^{i k(x-\xi)}\left\{k+u_{1}(\xi)-u_{1}(x)-2 \pi(x-\xi) u_{1}(x)+\frac{1}{k}\left[u_{1}(x) u_{1}(\xi)-u_{2}(x)-\right.\right.\right. \\
& -u_{2}(\xi)-i u_{1}(0)+\frac{i}{\pi}\left(u_{1}(\xi)-u_{1}(x)-u_{1}(\pi)++2 u_{1}(0)\right)+\frac{1}{\pi^{2}}+2 \pi(x-\xi) u_{1}(x)- \\
& \left.\left.-u_{1}(\xi) u_{1}(\pi)+2 \pi^{2}(x-\xi)^{2} u_{1}^{2}(\pi)\right]\right\}=\frac{i}{2}\left[u_{2}(x)+u_{1}(\xi) u_{1}(x)+u_{2}(\xi)+u_{i}(0)\right]+ \\
& +\frac{1}{2 \pi}\left\{F\left(e^{i(x-\xi)},-1\right)+\left(u_{1}(\xi)-u_{1}(x)-2 \pi(x-\xi) u_{1}(\pi) F\left(e^{i(x-\xi)}, 0\right) i\right.\right. \\
& +\left[u_{1}(x) u_{1}(\xi)-u_{2}(x)-u_{2}(\xi)-i u_{1}(0)++\frac{i}{\pi}\left(u_{1}(\xi)-u_{1}(x)-u_{1}(\pi)+2 u_{i}(0)\right)+\right. \\
& \left.\left.+\frac{1}{\pi^{2}}+2 \pi(x-\xi)\left(u_{1}(x)-u_{1}(\xi)\right) u_{1}(\pi)+2 \pi^{2}(x-\xi)^{2} u_{1}^{2}(\pi)\right] F\left(e^{i(x-\xi)}\right]\right\}
\end{aligned}
$$

(2) If $x<\xi$, then :

$$
\begin{aligned}
& \sum_{k=1}^{\infty}\left[\lambda_{k} \mathbf{H}_{k}(x, \xi)+\frac{1}{2 \pi} e^{-k(x-\xi)}\left\{k+u_{1}(x)-u_{1}(\xi)-2 \pi(x-\xi) u_{1}(\pi)+\right.\right. \\
&+ \frac{1}{k}\left[u_{1}(x) u_{1}(\xi)-u_{2}(x)-u_{2}(\xi)-i_{1}^{\prime}(0)+\frac{i}{\pi}\left(u_{1}(x)-u_{1}(\xi)-u_{1}(\pi)+\right.\right. \\
&\left.\left.\left.+2 u_{1}^{\prime}(0)\right)+\frac{1}{\pi^{2}}+2 \pi(x-\xi)\left(u_{1}(\xi)-u_{1}(x)\right) u_{1}(\pi)+2 \pi^{2}(x-\xi)^{2} u_{1}^{2}(\pi)\right]\right\} \\
&= \frac{i}{2}\left[u_{2}(x)+u_{1}(\xi) u_{1}(x)+u_{2}(\xi)+u_{1}^{\prime}(0)\right]+\frac{1}{2 \pi}\left\{F\left(e^{-1(x-\xi)},-1\right)+\left(u_{1}(x)-\right.\right. \\
&-\left.u_{1}(\xi)-2 \pi(x-\xi) u_{1}(\pi)\right) \cdot F\left(e^{l(x-\xi)}, 0\right)+\left[u_{1}(x) u_{1}(\xi)-u_{2}(x)-u_{2}(\xi)-i u_{1}^{\prime}(0)+\right. \\
&\left.\left.+\frac{1}{\pi}\left(u_{1}(x)-u_{1}(\xi)-u_{1}(\pi)+2 u_{1}^{\prime}(0)\right)+\frac{1}{\pi^{2}}+2 \pi(x-\xi)\left(u_{1}(\xi)-u_{1}(x)\right) u_{1}(x)+2 \pi^{2}(x-\xi)^{2} u_{1}^{2}(x)\right]\right\}
\end{aligned}
$$


3. Consider the Sturm-Louville problem

$$
\begin{aligned}
& -y^{\prime \prime}(x)+q(x) y(x)=\lambda y, \quad \lambda=\rho^{2}, \quad 0 \leq x \leq \pi \\
& y(0)=y\left(\frac{\pi}{2}\right)+y(\pi)=0,
\end{aligned}
$$

where $q(x)$ is a sufficiently smooth function defined on the interval $[0, \pi]$.

Upon using the definition of $\Delta(\rho)$ and the formulae (6.6), (6.7), (6.8), we have:

$$
\begin{gathered}
\Delta(\rho)=\frac{\sin \rho \pi+\sin \rho \frac{\pi}{2}}{\rho}+\frac{-i\left[u_{1}(\pi) \cos \rho \pi+u_{1}\left(\frac{\pi}{2}\right) \cos \rho \frac{\pi}{2}\right]}{\rho^{2}}+ \\
+\frac{-i\left[\left(i u_{2}(\pi)-u_{1}^{\prime}(0)\right) \sin \rho \pi+\left(i u_{2}\left(\frac{\pi}{2}\right)-u_{1}^{\prime}(0)\right) \sin \rho \frac{\pi}{2}\right]}{\rho^{3}}+ \\
+\frac{\left[2 u_{1}(\pi) u_{1}^{\prime}(0)-i u_{3}(\pi)\right] \cos \rho \pi+\left[2 u_{1}\left(\frac{\pi}{2}\right) u_{1}^{\prime}(0)-i u_{3}\left(\frac{\pi}{2}\right)\right] \cos \rho \frac{\pi}{2}}{\rho^{4}}+\ldots
\end{gathered}
$$

To find the eigenvalues of problem (6.24)-(6.25), we put

$$
\begin{gathered}
V=e^{-t^{\frac{\pi}{2}}}=\sum_{v=0}^{\infty} \frac{a_{v}}{\rho^{v}} ; \quad \Delta(\rho)=0 \text { then } \\
V^{4}\left[-\frac{1}{2 i \rho}-\frac{i u_{1}(\pi)}{2 \rho^{2}}+\frac{i\left[i u_{2}(\pi)-u_{1}^{\prime}(0)\right]}{3 i \rho^{3}}+\ldots\right]+V^{3}\left[-\frac{1}{2 i \rho}-\frac{i u_{1}\left(\frac{\pi}{2}\right)}{2 \rho^{2}}+\frac{i\left[i u_{2}\left(\frac{\pi}{2}\right)-u_{1}^{\prime}(0)\right]}{3 i \rho^{3}}+\ldots\right]+ \\
+V\left[\frac{1}{2 i \rho}-\frac{i u_{1}\left(\frac{\pi}{2}\right)}{2 \rho^{2}}+\frac{i\left[i u_{2}\left(\frac{\pi}{2}\right)-u_{1}^{\prime}(0)\right]}{3 i \rho^{3}}+\ldots\right]+\left[\frac{1}{2 i \rho}-\frac{i u_{1}(\pi)}{2 \rho^{2}}+\frac{i\left[i u_{2}(\pi)-u_{1}(0)\right]}{3 i \rho^{3}}+\ldots\right]=0,
\end{gathered}
$$

and

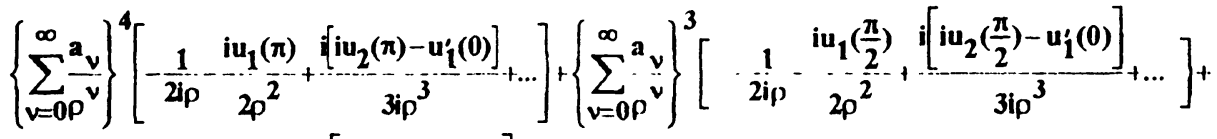

$+\left\{\sum_{v \rightarrow \infty}^{\infty} \frac{a_{v}}{\rho^{v}}\right\}\left[\frac{1}{2 i \rho}-\frac{i u_{1}\left(\frac{\pi}{2}\right)}{2 \rho^{2}}+\frac{i\left[i u_{2}\left(\frac{\pi}{2}\right)-u_{1}^{\prime}(0)\right]}{3 i \rho^{3}}+\ldots\right]+\left[\frac{1}{2 i \rho}-\frac{i u_{1}(\pi)}{2 \rho^{2}}-\frac{i\left[i u_{2}(\pi)-u_{1}^{\prime}(0)\right]}{3 i \rho^{3}}+\ldots\right]=0$

Equating the coeflicients of $\rho^{-k}(k=1,2,3, \ldots)$ to zero, we have

$$
-a_{0}^{4}-a_{0}^{3}+a_{0}+1=0
$$

Solving the Equation (6.29), we have

$$
a_{0}^{(1)}=1, \quad a_{0}^{(2)}=-1, \quad a_{0}^{(3)}=\frac{-1+i \sqrt{3}}{2}, \quad a_{0}^{(4)}=\frac{-1-i \sqrt{3}}{2}
$$

Upon using the result in [9], we have for the eigenvalues of the problem (6.24)-(6.25), the following asymptotic formula

$$
\rho_{k, s}-4 k-\frac{2}{i \pi} \ln a_{0}^{(s)}+\frac{a_{1}^{(s)}}{2 a_{0}^{(s)} k \pi}-\frac{a_{1}^{(s)} \ln a_{0}^{(s)}}{4 i a_{0}^{(s)} \pi k^{2}}+\ldots
$$

where $s=1,2,3,4$, and

$$
a_{1}^{(1)}=\frac{1}{6} u_{1}(\pi), a_{1}^{(2)}=-\frac{1}{2} u_{1}(\pi), a_{1}^{(3)} \cdots \frac{u_{1}(\pi)}{4\left(a_{0}^{(3)}\right)^{3}+3\left(a_{0}^{(3)}\right)^{2}-1}, a_{1}^{(4)} \frac{u_{1}(\pi)}{4\left(a_{0}^{(3)}\right)^{3}+3\left(a_{0}^{(3)}\right)^{2}-1},
$$


Using the formula (6.31), we have

where

$$
\rho_{k, s}^{2}=\sum_{v=0}^{\infty} \frac{Q_{v}^{(s)}}{k^{v-2}}
$$

$Q_{0}^{(s)}=16, Q_{1}^{(s)}=-16 i \ln a_{0}^{(s)}, Q_{2}^{(s)}=-\frac{4}{\pi^{2}}\left(\ln a_{0}^{(s)}\right)^{2}-\frac{4 a_{1}^{(s)}}{a_{0}^{(s)} \pi}, Q_{3}^{(s)}=\frac{-2 i}{\pi^{2}}(\pi-1) a_{1}^{(s)} \ln a_{0}^{(s)}, \ldots$

According to (4.1), we see that the Green's function $G(x, \xi, \rho)$ of the problem (6.24)-(6.25) has the following asymptotic formulae:

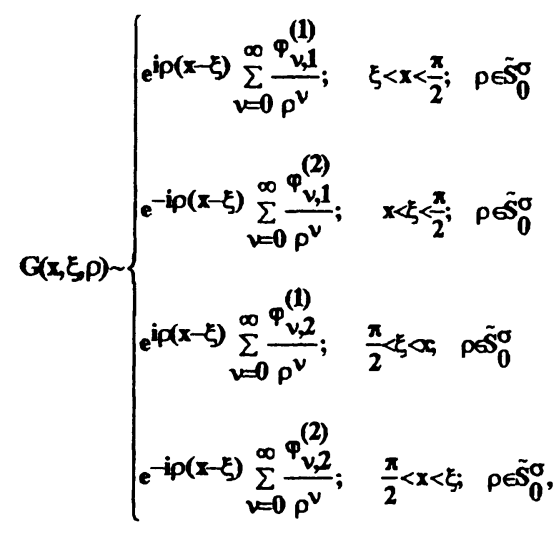

where

$$
\begin{aligned}
& \varphi_{0,1}^{(1)}=\varphi_{0,1}^{(2)}=\varphi_{0,2}^{(1)}=\varphi_{0,2}^{(2)}=0 \quad \varphi_{1,1}^{(1)}=\varphi_{1,1}^{(2)}=\varphi_{1,2}^{(1)}=\varphi_{1,2}^{(2)}=\frac{1}{21} \\
& \varphi_{2,1}^{(1)}=\varphi_{2,2}^{(1)}=-\varphi_{2,1}^{(2)}=-\varphi_{2,2}^{(2)}=\frac{1}{2}\left(u_{1}(x)-u_{1}(\xi)\right) \\
& \varphi_{3,1}^{(1)}=\varphi_{3,1}^{(2)}=\varphi_{3,2}^{(1)}=\varphi_{3,2}^{(2)}=\frac{i}{2}\left[u_{2}(x)+u_{1}(\xi) u_{2}(x)+u_{2}(\xi)+i u_{1}^{\prime}(0)\right]
\end{aligned}
$$

Upon using the asymptotic formulae (6.35) for $\mathbf{G}(x, \xi, \rho)$ in $\tilde{\mathbf{S}}_{0}^{\sigma}$ and the definition of the function $H_{k, s}(x, \xi)$, we have

$$
H_{k, s}(x, \xi)=\left\{\begin{array}{cc}
e^{i \rho_{k, s}(x-\xi)} \sum_{v=0}^{\infty} \frac{\phi_{v, 1}^{(3)}}{\rho_{k, s}^{v}} ; \quad \xi<x<\frac{\pi}{2} ; \rho_{k, s} \in \tilde{S}_{0}^{\sigma}, \\
e^{-i \rho_{k, s}(x-\xi)} \sum_{v=0}^{\infty} \frac{\phi_{v, 1}^{(4)}}{\rho_{k, s}^{v}} ; \quad \xi<x<\frac{\pi}{2} ; \rho_{k, s} \in \tilde{S}_{0}^{\sigma}, \\
e^{i \rho_{k, s}(x-\xi)} \sum_{v=0}^{\infty} \frac{\phi_{v, 2}^{(3)}}{\rho_{k, s}^{v}} ; & \frac{\pi}{2}<\xi<x ; \rho_{k, s} \in \tilde{S}_{0}^{\sigma}, \\
e^{-i \rho_{k, s}(x-\xi)} \sum_{v=0}^{\infty} \frac{\phi_{v, 2}^{(4)}}{\rho_{k, s}^{v}} ; & \frac{\pi^{0}<x<\xi ; \rho_{k, s} \in \tilde{S}_{0}^{\sigma},}{2}
\end{array}\right.
$$

where

$$
\phi_{0,1}^{(3)}=\phi_{0,1}^{(4)}=\phi_{0,2}^{(3)}=\phi_{0,2}^{(4)}=0 ; \quad \phi_{1,1}^{(3)}=\phi_{1,1}^{(4)}=\phi_{1,2}^{(3)}=\phi_{1,2}^{(4)}=-\frac{1}{2 \pi} ;
$$




$$
\begin{aligned}
& \phi_{2,1}^{(3)}=\phi_{2,2}^{(3)}=-\phi_{2,1}^{(4)}=-\phi_{2,2}^{(4)}=\frac{1}{2 \pi}\left(u_{1}(\xi)-u_{1}(x)\right) \\
& \phi_{3,1}^{(3)}=\phi_{3,2}^{(3)}=-\phi_{3,1}^{(4)}=-\phi_{3,2}^{(4)}=\frac{1}{2 \pi}\left[u_{2}(x)+u_{2}(\xi)-u_{1}(x) u_{1}(\xi)+\right. \\
& +i u_{1}^{\prime}(0)+\frac{i}{\pi}\left(u_{1}(x)+u_{1}(\pi)-u_{1}(\xi)-2 u^{\prime}(0)-\frac{1}{\pi^{2}}\right], \ldots
\end{aligned}
$$

From formulae (6.33) and (6.37), we get

$$
p_{k, s}^{2} H_{k, s}(x, \xi) \begin{cases}e^{-21(x-\xi)\left(2 k-\frac{1}{\pi} \ln a_{0}^{(s)}\right.} \sum_{n=0}^{\infty} \sum_{p=0}^{n} \sum_{\ell=0}^{p} \frac{\Psi_{n-p}^{(s)} Q_{\ell}^{(s)} \phi_{p-\ell, 1}^{(3)}}{k^{n-2}} & \xi<x<\frac{\pi}{2} \\ e^{21(x-\xi)\left(2 k-\frac{1}{\pi} \ln a_{0}^{(s)}\right.} \sum_{n=0}^{\infty} \sum_{p=0}^{n} \sum_{\ell=0}^{p} \frac{\Psi_{n-p}^{(s)} Q_{\ell}^{(s)} \phi_{p-\ell, 1}^{(4)}}{k^{n-2}} & x<\xi<\frac{\pi}{2} \\ e^{-21(x-\xi)\left(2 k-\frac{1}{\pi} \ln a_{0}^{(s)}\right.} \sum_{n=0}^{\infty} \sum_{n=0}^{n} \sum_{\ell=0}^{p} \frac{\Psi_{n-p}^{(s)} Q_{\ell}^{(s)} \phi_{p-\ell, 2}^{(3)}}{k^{n-2}} & \frac{\pi}{2}<\xi<x \\ e^{21(x-\xi)\left(2 k-\frac{1}{\pi} \ln a_{0}^{(s)}\right)} \sum_{n=0}^{\infty} \sum_{p=0}^{n} \sum_{\ell=0}^{p} \frac{\Psi_{n-p}^{(s)} Q_{\ell}^{(s)} \phi_{p-\ell, 2}^{(4)}}{k^{n-2}} ; & \frac{\pi}{2}<x<\xi\end{cases}
$$

where,...

$\Psi_{0}^{(s)}(x, \xi)=1, \quad \Psi_{1}^{(s)}(x, \xi)=\frac{i a_{1}^{(s)}(x-\xi)}{2 a_{0}^{(s)} \pi} ; \quad \Psi_{2}^{(s)}=-\frac{1}{8}(x-\xi)^{2}\left(\frac{a_{1}^{(s)}}{a_{0}^{(s)} \pi}\right)^{2}$,

$\Psi_{3}^{(s)}(x, \xi)=\frac{-\left(a_{1}^{(s)} \ln a_{0}^{(s)}\right)(x-\xi)}{\pi a_{0}^{(s)}}+\frac{i\left(\frac{a_{1}^{(s)}(x-\xi)}{2 a_{0}^{(s)} \pi}\right)^{3}}{6}, \ldots$

Upon using formulae (6.36), (6.38), (6.39) and (6.40), we have the following theorem :

\section{THEOREM 6.2}

1) If $\xi<x<\frac{\pi}{2}$, then the regularized sum of the first order for eigenfunctions of the problem

(6.24)-(6.25) are given by the followig formulae

$\sum_{k=1}^{\infty} \sum_{s=1}^{4}\left[\lambda_{k, s} H_{k, s}(x, \xi)-e^{1(x-\xi)\left(-4 k-\frac{2}{1 \pi} \ln a_{0}^{(s)}\right)} \sum_{n=0}^{3} \sum_{p=0}^{n} \sum_{l=0}^{p} \frac{\Psi_{n-1}^{(s)} Q_{l}^{(s)} \phi_{l, l, 1}^{(3)},}{k^{n-2}}\right]$.

$=\frac{i}{2}\left[u_{2}(x)+u_{1}(\xi) u_{1}(x)+u_{2}(\xi)+i u_{1}^{\prime}(0)\right]-\sum_{s=1}^{4} \sum_{n=0}^{3} \sum_{p=0}^{n} \sum_{\ell=0}^{p} e^{1(x-\xi)\left(-\frac{2}{i} \pi a_{0}^{(s)}\right)}$

.$F\left(e^{-41(x-\xi)}, n-2\right) \Psi_{n-p}^{(s)} Q_{\ell}^{(s)} \phi_{p-\ell, 1}^{(3)}$

(2) If $x<\xi<\frac{\pi}{2}$, then the regularized sum of the first order for eigenfunctions of the problem (6.24)-(6.25) are given by the followig formulae

$$
\begin{aligned}
& \sum_{k=1}^{\infty} \sum_{s=1}^{4}\left[\lambda_{k, s} H_{k, s}(x, \xi)-e^{-i(x-\xi)\left(-4 k-\frac{2}{i} \ln a^{(s)}\right)} \sum^{3} \sum_{n=0}^{n} \sum_{p=0}^{p} \frac{\Psi_{\ell=0}^{(s)} \frac{Q^{(s)} \ell^{(4)}}{p-\ell, 1}}{k^{n-2}}\right] \\
& =\frac{i}{2}\left[u_{2}(x)+u_{1}(\xi) u_{1}(x)+u_{2}(\xi)+i u_{1}^{\prime}(0)\right]-\sum_{s=1}^{4} \sum_{n=0}^{3} \sum_{p=0}^{n} \sum_{f=0}^{p} e^{-1(x-\xi)\left(-\frac{2}{i \pi} \ln a^{(s)}\right)}
\end{aligned}
$$




$$
\text { . } F\left(e^{4(x-\xi)}, n-2\right) \psi_{n-p}^{(s)} Q_{\ell}^{(s)} \phi_{p-\ell, 1}^{(4)}
$$

(3) If $\frac{\pi}{2}<\xi<x$, then the regularized sum of the first order for eigenfunctions of the problem (6.24)-(6.25) are given by the followig formulae.

$$
\begin{aligned}
& \sum_{k=1}^{\infty} \sum_{s=1}^{4}\left[\lambda_{k, s} H_{k, s}(x, \xi)-e^{I(x-\xi)\left(-4 k-\frac{2}{i \pi} \ln a_{0}^{(s)}\right)} \sum_{n=0}^{3} \sum_{p=0}^{n} \sum_{\ell=0}^{p} \frac{\Psi_{n-p}^{(s)} Q_{\ell}^{(s)}{ }_{\phi}^{(3)}-\ell, 2}{k^{n-2}}\right] \\
& =\frac{1}{2}\left[u_{2}(x)+u_{2}(\xi)+u_{1}(x) u_{1}(\xi)+i u_{1}^{\prime}(0)\right]-\sum_{s=1}^{4} \sum_{n=0}^{3} \sum_{p=0}^{n} \sum_{\ell=0}^{p} e^{I(x-\xi)\left(-\frac{2}{i \pi} \ln a^{(s)}\right)} . \\
& . F\left(e^{4(x-\xi)}, n-2\right) \Psi_{n-p}^{(s)} Q_{\ell}^{(s)} \phi_{p-\ell, 2}^{(3)}
\end{aligned}
$$

(4) If $\frac{\pi}{2}<x<\xi$, then the regularized sum of the first order for eigenfunctions of the problem (6.24)-(6.25) are given by the followig formulae

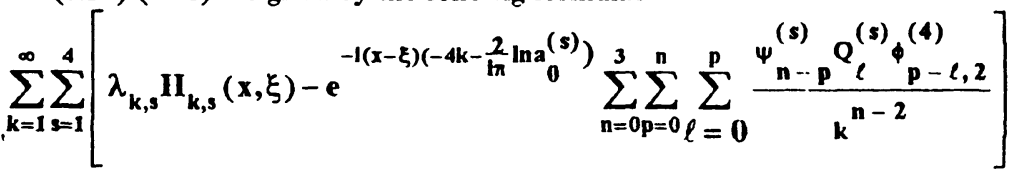

$$
\begin{aligned}
& =\frac{i}{2}\left[u_{2}(x)+u_{2}(\xi)+u_{1}(x) u_{1}(\xi)+i u_{1}^{\prime}(0)\right]-\sum_{s=1}^{4} \sum_{n=0}^{3} \sum_{p=0}^{n} \sum_{\ell=0}^{p} e^{1(x-\xi)\left(-\frac{2}{i \pi} \ln 2_{0}^{(s)}\right)} \\
& F\left(e^{4(x-\xi)}, n-2\right) \Psi_{n-p}^{(s)} Q_{\ell}^{(s)} \phi_{p-\ell, 2}^{(4)}
\end{aligned}
$$

\section{REFERENCES}

[1] SADOVNICHII V. A., Theory of Operators (Moscow University 1979-Moscow SSSR), 192-226. [Russian]. Translated from the second Russian edition by Roger cooke. Contemporary soviet Mathematics, consultants Bureau, New York, 1991.

[2] GELFAND 1. M., LEVITAN M. B., " On a simple identity for eigenvalues of a differential operator of the second order" Dokd Akad Nauk SSSR 88 (1953) 593-596 [ Russian]

[3] CHARLES J. A., HALBERG JR and KRAMER V. A."Ageneralization of the one trace concept "Duke Math. J. 27 (1960) 607-617.

[4] LIDSKY V. B., BADOVNICHII V.A. " Regularized sums of the roots of the class of integral functions" Dokl Akad. Nauk SSSR 176 No 2. (1967) 259-262. [Russian]

[5] LIDSKY V. B., BADOVNICHII V. A. " Regularized sums of the roots of the one class of integral functions." Functional analysis V. 1 № 2 (1967) 52-59 [Russian]

[6] LIDSKY V. B., SADOVNICHII V. A. " Formulae of traces in case of Orr-Sommerfield equation. Esv. Akad Nauk SSSR. ser. Math. V. 32 No 3(1968) 633-648. [Russian].

[7] SADOVNICHII V. A., LYUBISHKIN V. A. and BELABBASY U. " On regularized sums of the roots of integral function of some class ". Dokel Akad Nauk SSSR. V. 254, № 6 (1980). 1346-1348 [Russian] 
[8] BADOVNICHII V. A., LYUBIBHKIN V. A." Regularized sums of roots of class of integral functions of an exponential type". Dokl Akad. Nauk SSSR. V. 256, № 4 (1981) 794-798 [Russian]

[9] SALEH S. A. " Spectral properties of some class of non-self adjoint problem with multipoint conditions" PH. D. Moscow university (1984).[Russian].

[10] BADOVNICHII V. A." On the trace with weight and asymptotic spectral functions" Differential equations V. 10 No10 (1974) 1808-1818 [ Russian ]

[11] MARCHENKO V. A. " Sturm-Liouville operators and Applications" Translated from the Russian by A. Lacoh. Birkhauser Verlag. Basel. Boston-Stuttgart(1986) 51

[12] NAIMARK M. A. " Linear differential operators" Nauk Moscow (1969) [Russian]. Tanslated by E. R. Dawson, Queen's college, Dundee. English translation edited by W. N.Everitt Professor of Mathematics, Queen's College, Dundee.

[13] ' ERDELYI A., MAGNUS W., OBERHETTINGER F., and TRICOMI F. G. "Higher transcendental functions "V. 1 .New york-Toronto-London McGRAW-HLLL BOOK COMPANY, INC 1953 26-27.

[14] KLUSCH D." On the Taylor expansion of the Lerch-Zeta- function " Journal of Mathematical analysis and applications 170 , (1992) 513-523 


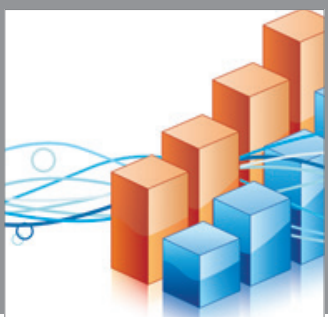

Advances in

Operations Research

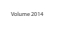

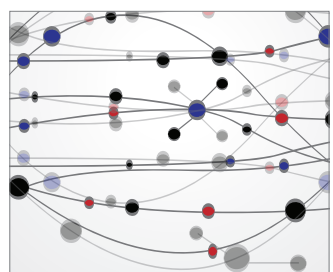

\section{The Scientific} World Journal
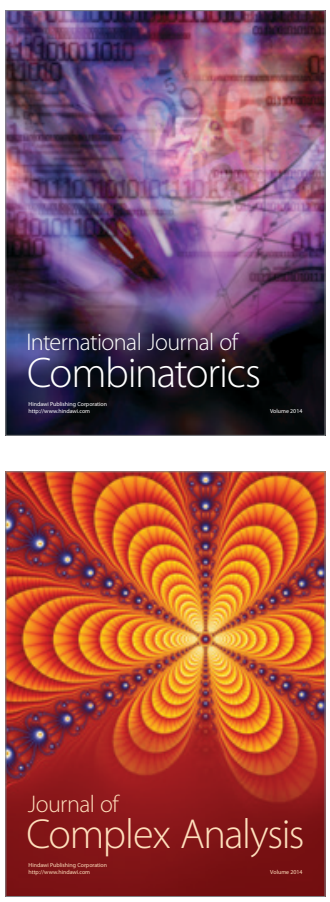

International Journal of

Mathematics and

Mathematical

Sciences
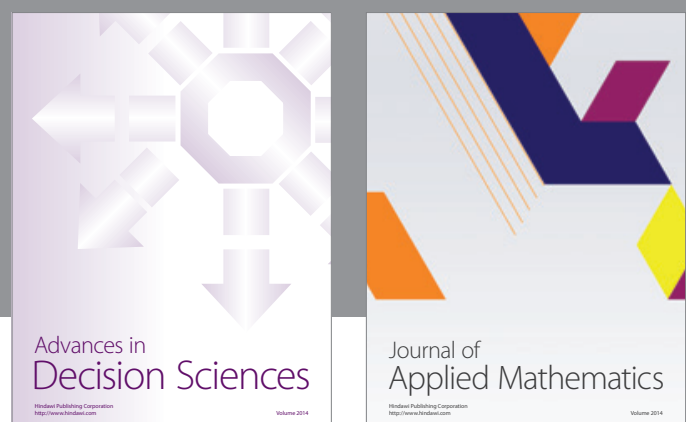

Journal of

Applied Mathematics
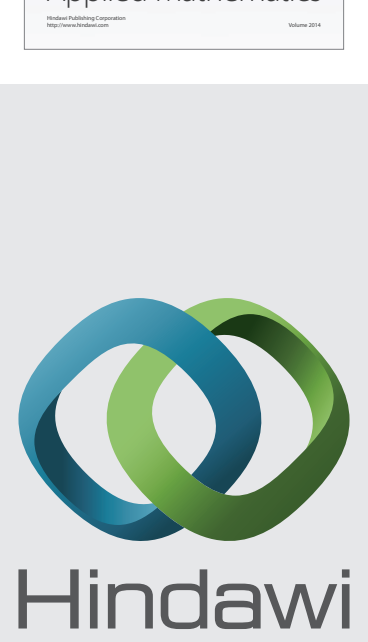

Submit your manuscripts at http://www.hindawi.com
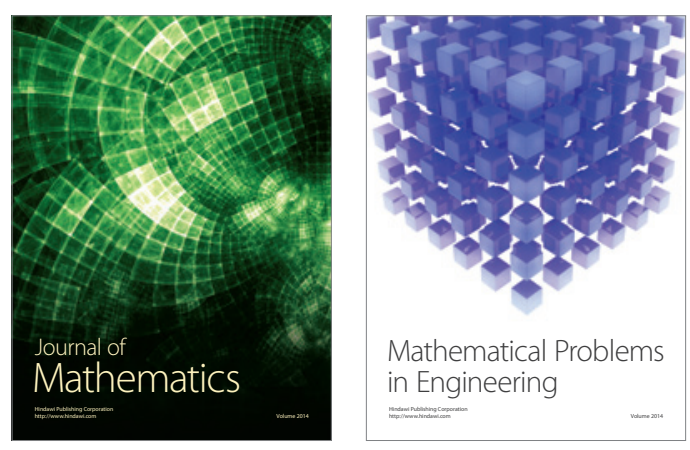

Mathematical Problems in Engineering
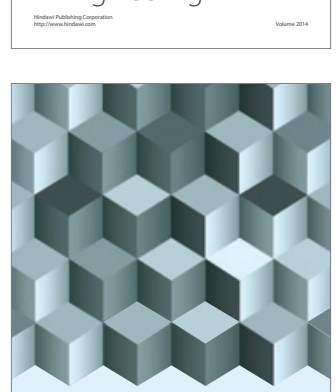

Journal of

Function Spaces
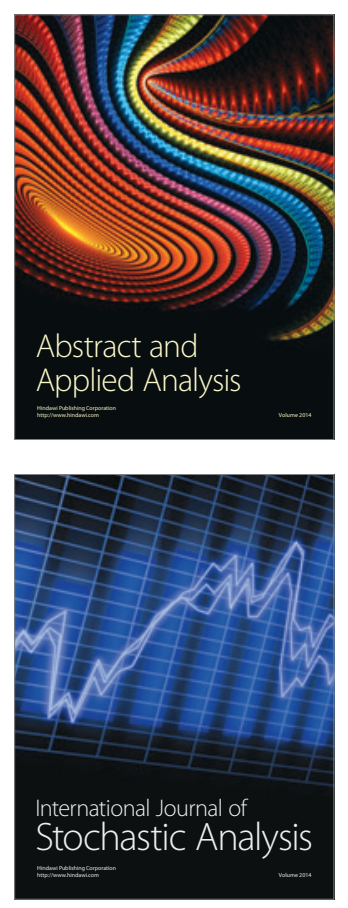

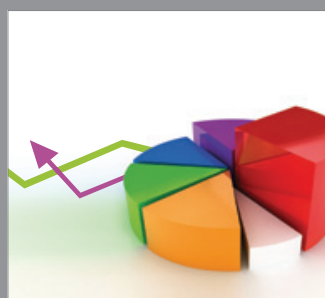

ournal of

Probability and Statistics

Promensencen
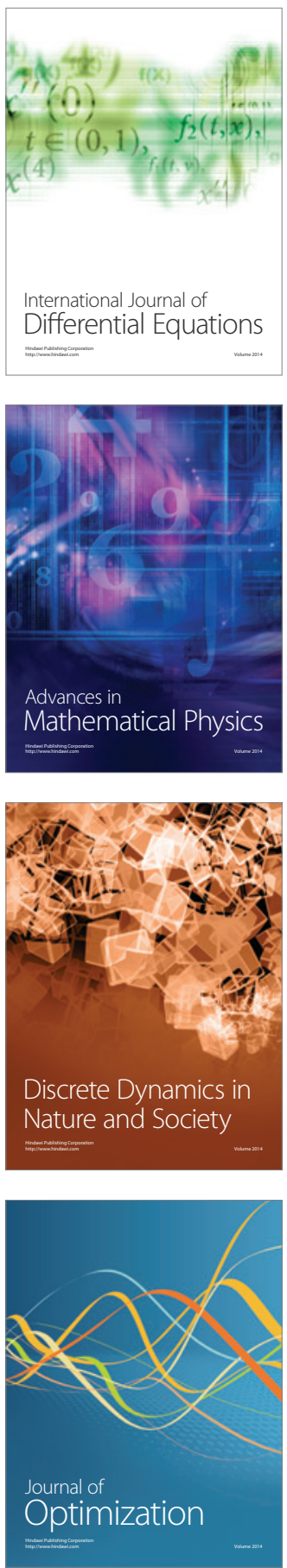